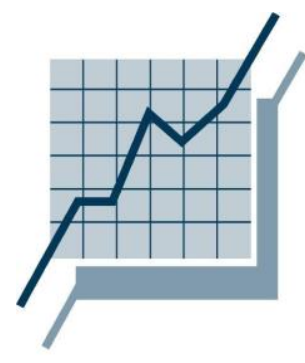

\title{
DECISIÓN DE LOCALIZACIÓN DEL HOGAR EN ESPAÑA. MODELO DE CLASES LATENTES VS. MODELO MIXTO.
}

\author{
ANASTASIA HERNÁNDEZ ALEMÁN* \\ Instituto Universitario de Turismo y Desarrollo Económico Sostenible (Tides). UNIVERSIDAD DE LAS \\ PALMAS DE GRAN CANARIA, ESPAÑA. \\ E-mail: anastasia.hernandez@ulpgc.es;
}

Recibido: 1 de Diciembre de 2018

Aceptado: 26 de Julio de 2019

\section{RESUMEN}

En este trabajo, se analiza la heterogeneidad inobservable en la elección de la zona de residencia del hogar, distinguiendo entre tres alternativas: zona urbana, zona interurbana y zona rural. La estructura más flexible del modelo de clases latentes permite profundizar en mayor medida en el análisis de la heterogeneidad inobservable respecto al más limitado análisis de la aleatoriedad de los parámetros del modelo logit mixto. Del análisis realizado se puede distinguir entre dos clases o grupos de hogares con comportamientos diferenciados en cuanto a la decisión de localización y, asociado a ello, con estilos de vida diferentes. Así, determinados atributos de la localización de la vivienda, como el ruido, la contaminación o la delincuencia, afectan a la preferencia del hogar de forma diferente según la clase de pertenencia. También las características individuales del responsable del hogar, como la edad, el género o el nivel educativo, tienen un impacto diferente en la preferencia por la localización según la clase de pertenencia. Los resultados permiten distinguir dos estilos de vida asociados a cada clase o grupo de preferencia: el suburbano y el urbano.

Palabras clave: localización, vivienda, estilos de vida.

\begin{abstract}
In this work the unobservable heterogeneity respect to the choice of household's residential location is analyzed, considering three alternatives: urban area, interurban area and rural area. It is employed latent class choice model to represent this behavior and the results are compared with the multinomial and mixed logit models. The more flexible structure of the latent class model allows us more deeply into the analysis of unobservable heterogeneity with respect to the more limited analysis of the randomness of the parameters of the mixed logit model. The empirical results indicate two classes or groups of households with differentiated behaviors regarding to the decision of location and associated to different lifestyles. Thus, certain attributes of the location of the dwelling, such as noise, pollution or delinquency, have an effect on household preference in a different way according to the class of belonging. Also, individual characteristics of the household's head, such as age, gender or educational level, have a different impact on the preference for location according to the class of belonging. The results allow us to distinguish two lifestyles associated with each class or group of preference: suburban and urban.
\end{abstract}

Keywords: location, housing, lifestyles.

Clasificación JEL: C35, R21. 


\section{INTRODUCCIÓN}

Gran parte de los trabajos que analizan la demanda de vivienda, en particular, en relación con su localización, se centran en los factores determinantes de los precios. Así, por ejemplo, encontramos en la literatura un extenso volumen de trabajos que emplean frecuentemente el método de los precios hedónicos. Menor es el volumen de trabajos con respecto al análisis de los factores socioeconómicos $\mathrm{y}$, en particular, en relación con los atributos que caracterizan la localización de la vivienda. Estos últimos factores afectan significativamente al proceso de decisión de la elección en relación con la ubicación de la vivienda. Asimismo, estos factores deben ser tenidos en cuenta en el marco de las políticas públicas de planificación territorial a efectos de alcanzar un uso más eficiente y racional en cuanto a uso del suelo residencial. Habitualmente los hogares más sensibles a la contaminación y al ruido suelen manifestar una mayor preferencia hacia la zona rural como mejor ubicación de la residencia. Ello aún disponiendo de un menor volumen de servicios públicos y privados; o incluso, a costa de asumir un mayor coste monetario y, también, en términos de tiempo en los desplazamientos diarios hacia el centro de trabajo. Otro proceso que se observa consiste en la mayor preferencia de los mayores por la zona urbana. Ello se explica probablemente por la facilidad de acceso a los servicios sanitarios. Por otro lado, paralelamente o a posteriori, los gestores públicos deben asumir el desarrollo de las infraestructuras públicas para dar satisfacción a las necesidades planteadas por esta demanda residencial. La mejora de las bases de microdatos, en particular, en España, aún con sus limitaciones en esta materia ${ }^{1}$, permite profundizar en algunos de estos factores cualitativos.

La mayor presión poblacional en torno a las ciudades junto con el proceso de envejecimiento demográfico al tiempo que un aumento de los flujos migratorios deriva en una mayor conflictividad social, y en mayores retos a abordar en la planificación territorial desde diferentes perspectivas (ambiental, social y económica). La transformación de los hábitats naturales, la generación de residuos por la acción humana, la búsqueda de mejores condiciones de vida, así como, el progresivo envejecimiento demográfico, afectan particularmente a los procesos urbanizadores.

España no queda al margen de esta tendencia hacia una mayor concentración de la población en las zonas urbanas. Conforme a los últimos datos proporcionados por el Banco Mundial, España ha pasado del 56,56\% de población residente en zonas urbanas en 1960 al 80,08\% en 2017. En el resto de Europa también se aprecia esa misma tendencia hacia la concentración de la población en zonas urbanas, pasando del 61,20\% en 1960 al 75,45\% en 2017 (Fig. 1). La distribución de la población española según el tamaño del municipio es reflejo de esta tendencia hacia una mayor concentración de la población en los grandes municipios. Conforme al Censo de Población y Viviendas de 2001, el 64,94\% de la población reside en municipios de más de 20.000 habitantes mientras que sólo el $15,07 \%$ de la población lo hace en municipios de 5.000 o menos habitantes. Esa diferencia porcentual ha aumentado en el último Censo de Población y Viviendas de 2011, de forma que el 68,04\% de los habitantes españoles reside en municipios de más de 20.000 habitantes $(+3,1 \%)$ mientras que el $12,74 \%(-2,33 \%)$ reside en municipios de 5.000 o menos habitantes. Además, esta diferencia porcentual está acompañada de un mayor envejecimiento de la población en torno a las ciudades y de una mayor presencia de extranjeros residentes los cuales han pasado del $71,71 \%$ al $73,76 \%$ en los municipios de más de 20.000 habitantes. Al mismo tiempo, disminuye la presencia de extranjeros en los municipios de 5.000 o menos habitantes, pasando del 9,36\% en 2001 al más reciente $8,53 \%$ en 2011.

La Encuesta de Condiciones de Vida (ECV, EU-SILC) también es reflejo de esta tendencia. Así, en el periodo que va de 2004 a 2007, el 47,27\% de los hogares españoles encuestados reside en zona urbana; mientras que, en 2017 ese porcentaje aumenta al 51,65\% (Hernández y León, 2018: 39). Paralelamente, los hogares residentes en zona rural han descendido desde el 32,10\% de media al más reciente $26,92 \%$ durante el mismo periodo de tiempo. Por otra parte, y a pesar de la reducción del tamaño del hogar, sigue predominando la vivienda de tamaño grande (cinco o más de cinco habitaciones), según su tipología. Así, por ejemplo, en la zona rural, el piso representa el 29,37\% de

\footnotetext{
${ }^{1}$ Datos de corte transversal y con muchas limitaciones con respecto a los atributos que caracterizan la localización y las propias características de la vivienda.
} 
los que un $18,02 \%$ son de tamaño grande frente al $11,35 \%$ restante. En la zona interurbana, el piso representa el $63,28 \%$ de los que un $37,22 \%$ son de tamaño grande.

Figura 1: Población urbana (\% del total).

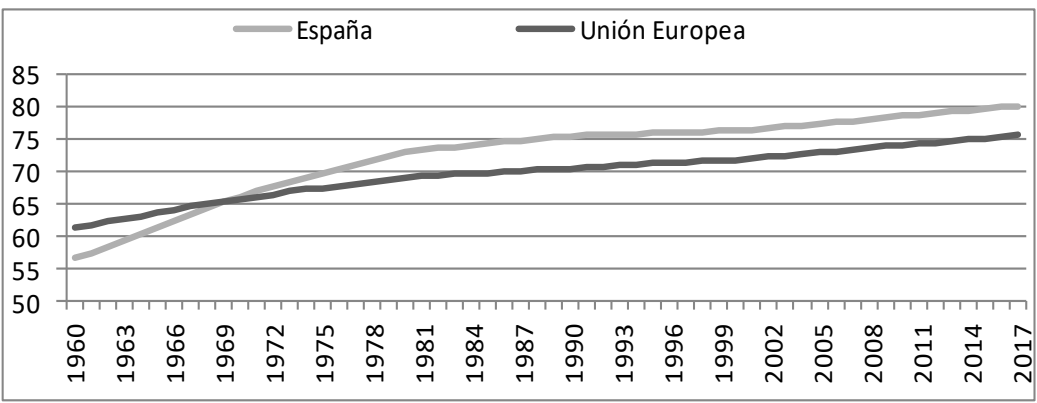

Fuente: Banco Mundial, 2018.

En la zona urbana, el piso representa el $87,29 \%$ de los que un $48,45 \%$ son de tamaño grande ${ }^{2}$. Ello se debe muy probablemente al lento proceso de ajuste por el lado de la oferta inmobiliaria en relación con la demanda.

Este trabajo es un análisis empírico que tiene por objetivo explicar el comportamiento del hogar en la elección de la localización de la vivienda desde el punto de vista de la zona urbana, interurbana o rural a partir de la preferencia revelada. Este comportamiento tiene impactos, entre otros, en el uso del suelo, en la demanda asistencial, en los nuevos usos del espacio en el contexto urbano y rural, y un largo etcétera. Para realizar el análisis, se busca el modelo de elección discreta que mejor se ajuste a los datos disponibles entre los modelos más frecuentemente empleados en la elección de vivienda residencial, aportando nueva evidencia en cuanto al modelo de clases latentes del logit condicionado. Hasta el momento, las aplicaciones empíricas del modelo de clases latentes en este ámbito son muy escasas. Prácticamente se reducen al trabajo de Walker et al., (2007). La estructura más flexible del modelo de clases latentes permite profundizar en mayor medida en el análisis de la heterogeneidad inobservable respecto al más limitado análisis de la aleatoriedad de los parámetros del modelo logit mixto. No obstante, como ya apuntara Hess (2014: 317): "La evidencia en estas comparaciones empíricas es variada, remarcándose que ambos modelos tienen sus ventajas y que la elección de la estructura más apropiada dependerá de los datos con los que se trabaje”.

El análisis empírico que se realiza en este trabajo se fundamenta teóricamente en el enfoque clásico de McFadden (1978). No obstante, el modelo presentado se nutre de las aportaciones más recientes en dos ámbitos. Por una parte, en el ámbito empírico, respecto de los factores determinantes de la elección de la localización de la vivienda; y, por otra parte, en el ámbito teórico, con respecto a la modelización mediante el uso del modelo de clases latentes del logit condicional. En este análisis empírico, las alternativas tienen que ver con el aspecto territorial (urbano, interurbano y rural) pero no se distingue respecto de la forma de tenencia (compra, alquiler). Ello porque el tamaño de la muestra respecto a la opción de alquiler no es significativo en España, aunque se observa una tendencia creciente en el tiempo con el más reciente 14,48\% en 2017. El objetivo de este trabajo no es describir el estilo de vida de cada clase o grupo de preferencia, pero sí evidenciar que cada clase o grupo de preferencia responde a un comportamiento diferente con respecto a la localización de la vivienda y ello se traduce en un estilo o segmento de vida diferente. Siguiendo a Walter y Li (2007) se distinguen dos estilos de vida asociados a cada clase o grupo de preferencia. Uno, el suburbano, de menor renta, de menor nivel educativo, con mayor frecuencia del tamaño de hogar más pequeño, con mayor frecuencia de la mujer como cabeza de familia; y otro, el urbano con mayor poder adquisitivo, con mayor nivel educativo, con más presencia del varón como cabeza de familia y con mayor frecuencia del tamaño de hogar superior a tres miembros.

El resto del artículo se distribuye como sigue. En el siguiente apartado, se realiza una breve revisión de la literatura respecto de la elección de la localización de la vivienda. En el tercer apartado, se expone la metodología. En el cuarto, se analizan los datos empleados, así como, los principales resultados de las estimaciones. En el apartado quinto, se recogen las conclusiones. Finalmente, el

\footnotetext{
${ }^{2}$ ver Tabla 2 del apartado 4.
} 
artículo termina con las referencias bibliográficas, y un apéndice en el que se definen las variables empleadas en los modelos estimados.

\section{ANTECEDENTES}

En esta área predomina el trabajo empírico muy vinculado a la formulación de políticas por lo que es difícil encontrar un marco teórico y empírico como única referencia (King, 2009; Clapham, 2018). Es por ello que en este apartado se realiza un breve recorrido de la literatura acerca de los diferentes enfoques en el tratamiento de la demanda de la vivienda. El análisis parte del modelo teórico de McFdden (1978) basado en la maximización de la utilidad de la elección de localización de la vivienda. Modelo que en este caso se aplica al hogar privado ${ }^{3}$, tomando como referencia la preferencia revelada. Los determinantes básicos que se analizan tienen que ver con la renta permanente del hogar y con otras características socioeconómicas, así como, también, con los atributos ambientales de la localización de la vivienda.

Los modelos de elección residencial se formalizan inicialmente desde el punto de vista teórico con el trabajo de McFadden (1978). El principal inconveniente con el que tropieza este primer acercamiento de la teoría del consumidor desde el punto de vista económico a la elección de la localización de la vivienda es el incumplimiento de la hipótesis de independencia entre las alternativas prevista para el modelo logit multinomial. Para salvar este problema se tratan las unidades de vivienda individuales como las alternativas básicas entre las cuales se hace la elección. Cada unidad contiene un conjunto de atributos observables y no observables lo suficientemente amplio y rico al que responde cada individuo. Cada unidad física de vivienda está representada por un único punto en el espacio de los atributos. Por otro lado, el individuo puede percibir que dos viviendas tienen características similares en algunos atributos. Esta percepción es la que se modeliza. La vivienda es tratada como un conjunto de características que proporcionan utilidad. A partir de este enfoque clásico, encontramos hoy día una abundante literatura en la que se plantean enfoques alternativos, más flexibles y complejos para explicar la decisión de vivienda desde diferentes perspectivas. Siguiendo a Meen (2016) podemos distinguir un amplio volumen de trabajos en relación con la demanda de vivienda: los modelos hedónicos que son los más abundantes en la literatura (Brown et al., 1982; Bover et al., 2001; García y Raya, 2011; Ottensmann et al., 2008), los modelos espaciales (Leishman, 2009), los modelos de segregación (González y Ortega, 2013), los modelos de vecindad o barrio (Galster et al., 2010), los modelos de tenencia (Bourassa, 1995; Colom y Molés, 1999; Rodríguez y Barrios, 2003; 2007; Barrios et al., 2008), los modelos de duración y localización (Smith y Mertz, 1980), y los modelos dinámicos de transición (Zhang, 2004), entre otros. El sistema de preferencias respecto a los atributos de la vivienda y a otros factores determina la elección de la vivienda, siendo la localización en el territorio, generalmente en el ámbito urbano, una de las características esenciales al proceso de decisión.

En relación con las preferencias, y en una etapa más reciente, los estudios se centran en analizar cómo afectan los cambios en el ciclo de vida en la elección de la localización de la vivienda. El progresivo envejecimiento de las estructuras familiares, la disminución del tamaño del hogar, así como, las nuevas formas de familia (monoparentales con o sin hijos) son factores que tienen implicaciones directas en la demanda de vivienda y en la configuración de las nuevas ciudades (Dewilde, 2008; Bramley y Watkins, 2016). La adecuación o no de los recursos para atender las necesidades de los mayores en el seno del hogar propicia, en muchas ocasiones, un cambio en la elección de la vivienda y su localización (Bell et al., 2012; Castles, 1998; Ong et al., 2015). La nueva distribución de la edad en la formación de los hogares condiciona la demanda de vivienda (Ermisch, 1996). Así, por ejemplo, Mankiw y Weil (1989), Monnet y Wolf (2017), Myers (1990) y Faubert et al. (2015) argumentan que la compra de vivienda se corresponde con una edad específica (frecuentemente, de entre 20 a 49 años) lo que determina las características de la demanda de vivienda. Beer et al., (2011) distinguen tres periodos de transición en la vida del individuo en relación con la

\footnotetext{
${ }^{3}$ Se define el hogar privado como la persona o conjunto de personas que ocupan en común una vivienda familiar principal o parte de ella y consumen y/o comparten alimentos u otros bienes con cargo a un mismo presupuesto.
} 
vivienda. La primera ocupa hasta los 35 años. La segunda es la etapa de la consolidación entre los 35 y los 54 años y, por último, la etapa de la vejez. Se observa en algunas ciudades como, por ejemplo, Australia una creciente transición desde el abandono de la propiedad en el caso de los mayores hacia la incorporación a los programas de asistencia de vivienda, convirtiéndose en inquilinos de largo plazo. Buzar et al., (2007) analizan las transformaciones que han experimentado los centros de ciudad (Leipzig en Alemania, Ljubljana en Slovenia, Bolonia en Italia y León en España) a consecuencia de lo que llaman "segunda transición demográfica" la cual se traduce en nuevas relaciones familiares derivadas fundamentalmente del aumento de los divorcios, menor volumen de matrimonios y más tardíos, descenso de la natalidad, envejecimiento de la población, aumento de la edad de maternidad, reducción del tamaño de los hogares, entre otras. Estos autores observan un incremento de la concentración de estructuras familiares no tradicionales en los centros de ciudad. En Francia, por ejemplo, las nuevas poblaciones del centro de ciudad han estado marcados por un rápido crecimiento en el número de solteros y por la cohabitación de jóvenes profesionales (Ogden y Hall, 2000). Las nuevas estructuras familiares con mayor crecimiento de divorciados y separados propician una transición de propietarios a "ocupantes" (Hendershott et al., 2009). Por su parte, Dura-Guimera (2003) observa para la ciudad de Barcelona que algunos desarrollos estuvieron acompañados de profundos cambios en la composición socio-espacial del sistema urbano. Los cambios demográficos conducen a respuestas de demanda muy heterogéneas para los diferentes atributos de la vivienda, proporcionando información en cuanto a las características cualitativas de la demanda de vivienda (Eichholtz y Lindenthal, 2014). De los antecedentes en la literatura en materia de decisión de localización de la vivienda, el más cercano al análisis empírico que se realiza en este trabajo es el análisis de Walker y Li (2007). Estos autores emplean un modelo de clases latentes a efectos de analizar las preferencias con respecto a la decisión de localización de la vivienda, obteniendo tres segmentos o grupos de preferencias: residentes suburbanos, residentes urbanos y residentes de tránsito. En el trabajo de Walker y Li (opus cit.), las alternativas se distinguen, según sea la compra o el alquiler de la vivienda $\mathrm{y}$, según se trate de vivienda unifamiliar o en edificio.

\section{METODOLOGÍA,}

En el modelo teórico de McFadden (1978) el consumidor racional elegirá un lugar de residencia, teniendo en cuenta el peso de los diferentes atributos que componen esa elección respecto de las alternativas, así como, también, teniendo en cuenta las diferentes características socioeconómicas individuales o familiares, eligiendo aquella alternativa que maximice su utilidad. Así pues, tomando como referencia este enfoque teórico, se plantean tres modelos de elección discreta. Conforme al paradigma de la maximización de la utilidad aleatoria en la elección de alternativas en cuanto a elección de la localización de la vivienda (McFadden, 1978; McFadden y Train, 2000), el hogar $i$ elegirá la alternativa $j$ de entre $J$ alternativas si la utilidad de $j$ es mayor respecto de la alternativa base, lo que se expresa en la siguiente ecuación (1):

$$
V_{i j}=\beta_{i j}^{\prime} X_{i j}+\varepsilon_{i j} \quad i=1, \ldots, N \text { hogares } j=1, \ldots, J \text { alternativas }
$$

Donde $V_{i j}$ representa la utilidad proporcionada al hogar $i$ respecto de la elección de la alternativa $j$; $X^{\prime}$ es el vector de variables exógenas u observables. $B^{\prime}$ es el vector de parámetros desconocido asociado a las características socioeconómicas y/o a los atributos de las alternativas. El primer sumando de la ecuación es determinista. $\varepsilon^{\prime}$ es el vector aleatorio el cual sigue una función de distribución logística (iid)_valor extremo. El factor aleatorio, o las características inobservables, tendrá una función de distribución condicionada a las características observables.

En un modelo de probabilidad logit multinomial (MNL), la probabilidad de que el hogar $i$ elija la alternativa $j$ viene definida por la siguiente expresión:

$$
P_{i j}=\frac{\exp \left(\beta_{i j}^{\prime} X_{i j}\right)}{\sum_{k=1}^{J} \exp \left(\beta_{i k}^{\prime} X_{i k}\right)} j=1, \ldots, J ; i=1, \ldots . ., N
$$


En los últimos años se han realizado grandes avances en los modelos de elección discreta a efectos de flexibilizar la hipótesis de la Independencia de las Alternativas Irrelevantes (IAI) en el modelo logit multinomial (MNL) que en contadas ocasiones se verifica. Así, el modelo logit mixto (MLM) permite relajar esta hipótesis, de forma que la función de utilidad cambiaría a esta otra forma algo más flexible con respecto a la anterior de la ecuación (1):

$$
V_{i j}=\alpha^{\prime} X_{i j}+\mu_{i}^{\prime} Z_{i j}+\varepsilon_{i j}(3) \text {, }
$$

donde $\mathrm{X}_{\mathrm{ij}}$ y $\mathrm{Z}_{\mathrm{ij}}$ son vectores de las variables observadas en relación con las alternativas, $\alpha$ es un vector de coeficientes fijos, $\mu$ es un vector de términos aleatorios de media cero; $\varepsilon_{i j}$ es iid valor extremo. En este modelo, la proporción de utilidad inobservada (aleatoria) se corresponde con la expresión: $\mu_{i} Z_{i j}+\varepsilon_{i j}$, la cual admite correlación entre las utilidades derivadas de las diferentes alternativas. Los coeficientes que representan las preferencias pueden variar entre los individuos. El modelo es una generalización del MNL:

$$
P_{i j t}=\frac{\exp \left(\alpha^{\prime} X_{i j t}+\mu^{\prime} Z_{i j t}\right)}{\sum_{k=1}^{J} \exp \left(\alpha^{\prime} X_{i k t}+\mu^{\prime} Z_{i k t}\right)} j=1, \ldots, J ; i=1, \ldots, N
$$

Donde $X_{i j t}$ es un vector de características específicas individuales y atributos específicos de la alternativa en la observación $t$ y estimado con parámetros fijos; $Z_{i j t}$ es un vector de características específicas individuales y atributos específicos de la alternativa en la observación $t$ y estimado con parámetros aleatorios.

La otra forma alternativa que se plantea en este trabajo para flexibilizar la hipótesis IIA es el modelo logit condicional de clases latentes (LCCLM), pudiendo además distinguir entre clases o grupos en la muestra de acuerdo a las preferencias en cuanto a la elección de vivienda según la localización. En este caso, la probabilidad de que el hogar $i$ de entre $N$ hogares elija la alternativa $j$ de entre $J$ alternativas, condicionado a la pertenencia a la clase $s$ de entre un conjunto de $C$ clases que se corresponden $\operatorname{con} \beta$ parámetros de preferencias y $\theta$ parámetros de pertenencia a clase o grupo (ec.(6) y ec.(7)), es el resultado de la suma del siguiente producto (ec. (5)):

$$
\ln L(\beta, \theta)=\sum_{i=1}^{N} \ln \sum_{s=1}^{C} \pi_{s i}(\theta) P_{i}\left(\beta_{s}\right)
$$

Donde:

$$
\begin{gathered}
\pi_{s i}(\theta)=\frac{\exp \left(\theta_{s} z_{i}\right)}{1+\sum_{l=1}^{C-1} \exp \left(\theta_{l} z_{i}\right)} \\
P_{i}\left(\beta_{s}\right)=\prod_{j=1}^{J}\left\{\frac{\exp \left(\beta_{s} X_{i j}\right)}{\sum_{k=1}^{J} \exp \left(\beta_{s} X_{i k}\right)}\right\}^{y_{i j}}
\end{gathered}
$$

Donde $y_{i j}$ es la variable binaria que toma el valor 1 si el hogar $i$ elije la alternativa $j$. Cada alternativa es descrita por los atributos propios de cada alternativa y por las características específicas de cada hogar, $x_{i j}$. En este caso, sólo hay un escenario posible porque se trabaja con datos de corte transversal para un solo año (Pacífico y Yoo, 2013).

La diferencia entre MLM y LCCLM es que en este último los parámetros varían en función de la clase, y al tiempo son fijos dentro de cada clase. Varias son las aplicaciones empíricas realizadas a efectos de probar la validez de estos métodos de estimación más flexibles (Persson, 2002; Shen, 2009). 
En este caso, y tomando como referencia el valor log-likelihood de los modelos estimados ${ }^{4}$, así como aplicando el test previsto en Shen (2009:6) $\left(\rho^{2}\right)$, los resultados indican que LCCLM se ajusta mejor a los datos que el MNL o el MLM. En primer lugar, se estima el MNL comprobando que si eliminamos una alternativa, el coeficiente de algunas variables como la tenencia de hijos en el hogar varía ligeramente. Así pues, ya se puede intuir que no se verifica el test de la Independencia de las Alternativas Irrelevantes. En cuanto al LCCLM el número de clases se decide en función de los criterios $^{5}$ CAIC y BIC (Tabla 1). De entre las cinco clases posibles que se estiman, las mejores opciones son dos o tres clases, teniendo en cuenta los criterios CAIC y BIC. En el trabajo de Walker et al., (2007) con datos para 507 individuos tomados en base a un experimento de preferencias declaradas, resultan dos clases como la mejor opción tomando como referencia el criterio BIC, y cuatro clases tomando como referencia el criterio AIC. En este caso, la opción de dos clases es la que presenta menor valor por lo que el modelo LCCLM se estima para dos clases de preferencias.

Tabla 1: Estimación del no de clases del LCCLM

\begin{tabular}{ccccc}
\hline Clases & LLF & Nparam & CAIC & BIC \\
\hline 2 & $-11.561,48$ & 51 & $23.658,73$ & $23.607,73$ \\
3 & $-11.504,68$ & 77 & $23.818,27$ & $23.741,27$ \\
4 & $-11.467,39$ & 103 & $24.016,83$ & $23.913,83$ \\
5 & $-11.449,71$ & 129 & $24.254,60$ & $24.125,60$
\end{tabular}

Nota: LLF=Log-Likelihood Function; Nparam=nํ de parámetros; CAIC= Consistent Akaike's information criterion based on loglikelihood; $\mathrm{BIC}=$ Bayesian Information Criterion based on log-likelihood.

\section{DATOS Y RESULTADOS DE LA ESTIMACIÓN}

La aplicación empírica se realiza en el marco de la elección de la localización de la vivienda residencial según el tipo de zona urbana, interurbana y rural. El objetivo es determinar cómo afectan las características individuales del responsable del hogar, las características socioeconómicas del hogar, así como, los atributos propios de la zona, en la elección de la localización de residencia. Este trabajo se propone analizar la existencia de grupos o clases heterogéneas en cuanto a las preferencias en la elección de la localización de la vivienda. La base de microdatos consta de 13.740 hogares, 29.294 adultos y un total de 34.911 personas entre adultos y niños correspondientes a la Encuesta de Condiciones de Vida de 2017. A efectos de la estimación de los modelos se eliminan los hogares correspondientes a Ceuta y a Melilla por cuanto sólo cuentan con una alternativa de localización de la vivienda cual es la zona urbana. Los modelos son estimados para una muestra total de 13.430 hogares. Las características de la muestra se encuentran resumidas en la Tabla 2. La renta media más elevada se encuentra en la zona urbana y la más baja en la zona rural. Las medias de edad más altas se observan en la zona rural y en la zona urbana. El ruido, la contaminación y la delincuencia afectan en mayor medida a la zona urbana y, en menor porcentaje, a la zona rural. Según la tipología de la vivienda, el piso es más frecuente en la zona urbana $(87,29 \%)$, y la vivienda unifamiliar, lo es en la zona rural $(70,63 \%)$. Las viviendas de mayor tamaño según el número de habitaciones ocupan mayor porcentaje en la zona rural (73\%). Los hogares con niños presentan mayor porcentaje en la zona interurbana (38\%) respecto de las zonas urbana y rural con un $32 \%$ respectivamente. Se observa una diferencia según el género, de forma que en la zona rural predomina el cabeza de familia varón $(63,64 \%)$; mientras que, la cabeza de familia mujer es más frecuente en la zona urbana (42,38\%). El nivel educativo inferior presenta mayor porcentaje en la zona rural $(83,65 \%)$. La principal ventaja del análisis a partir de las preferencias reveladas frente a los experimentos diseñados con preferencias declaradas, además de superar las restricciones presupuestarias, es contar con una muestra de hogares amplia.

De los resultados obtenidos de los modelos estimados (Tablas 3 y 4), se verifica la significación de algunas variables en la preferencia respecto a la localización de la vivienda. La renta es una variable altamente significativa en todos los modelos estimados. En términos generales, a mayor renta la preferencia se inclina hacia la residencia en zona urbana. Esta preferencia es igual en ambas clases del LCCLM pero diferente en relación con las alternativas consideradas. Así, la clase 1 o el grupo de

\footnotetext{
${ }^{4}$ Ver Tabla 3.

${ }^{5}$ Se elige la opción con menos valor.
} 
hogares perteneciente a la clase 1 de preferencias es sensible a las dos alternativas: interurbana y rural, en relación con la alternativa urbana. Mientras que, el grupo de hogares de la clase 2 de preferencias sólo es sensible a la alternativa rural en relación con la urbana. La clase 2 de preferencias, y en relación a la zona interurbana, no parece ser sensible a casi ninguna de las variables consideradas, salvo con respecto al tamaño del hogar.

Tabla 2: Estadística descriptiva_ECV_2017 (EU_SILC)

\begin{tabular}{|c|c|c|c|}
\hline Variable & Zona urbana & Zona interurbana & Zona rural \\
\hline Hogares & 7.097 & 2.944 & 3.699 \\
\hline Edad media & 58,24 & 56,71 & 59,43 \\
\hline Renta media (+/-) & $31.333,59$ & $28.192,25$ & $24.424,96$ \\
\hline Renta media (solo +) & $31.412,02$ & $28.213,84$ & $24.477,68$ \\
\hline \multicolumn{4}{|l|}{ Ruido: $\%$} \\
\hline Sí & 18,84 & 12,91 & 8,06 \\
\hline No & 81,16 & 87,09 & 91,94 \\
\hline \multicolumn{4}{|l|}{ Contaminación: \% } \\
\hline - & 10,74 & 6,01 & 3,76 \\
\hline No & 89,26 & 93,99 & 96,24 \\
\hline \multicolumn{4}{|l|}{ Delincuencia: $\%$} \\
\hline 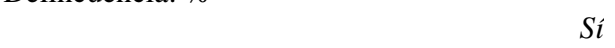 & 12,32 & 5,94 & 3,95 \\
\hline No & 87,68 & 94,06 & 96,05 \\
\hline \multicolumn{4}{|l|}{ Tipo de vivienda: $\%$} \\
\hline Unifamiliar & 12,71 & 36,72 & 70,63 \\
\hline piso & 87,29 & 63,28 & 29,37 \\
\hline \multicolumn{4}{|l|}{$\mathrm{N}$ habitaciones (dummy): \% } \\
\hline $0=1,2,3$ y 4 habitaciones & 42,35 & 36,37 & 27,17 \\
\hline $1=5,6$ y más habitaciones & 57,65 & 63,63 & 72,83 \\
\hline \multirow{3}{*}{\multicolumn{4}{|c|}{$\begin{array}{l}\mathrm{N} \text { habitaciones*tipo de vivienda: } \% \\
0=\text { dos posibilidades: vivienda unifamiliar (sea } \\
\text { de } 4 \text { o menos habitaciones, o bien, de más de } 5 \\
\text { habitaciones) y piso de menos de cuatro } \\
\text { habitaciones }\end{array}$}} \\
\hline & & & \\
\hline & & & \\
\hline $1=$ piso de cinco o más habitaciones & 48,45 & 37,22 & 18,02 \\
\hline Hogares con niños: $\%$ & 32,78 & 38,46 & 32,36 \\
\hline Nativo: $\%$ & 89,59 & 90,37 & 93,50 \\
\hline Extranjero: $\%$ & 10,41 & 9,63 & 6,50 \\
\hline \multicolumn{4}{|l|}{ Tamaño hogar: $\%$} \\
\hline $0=$ Hogares de entre 4 a 13 miembros & 22,29 & 27,07 & 24,20 \\
\hline $1=$ Hogares de entre 1 a 3 miembros & 77,71 & 72,93 & 75,80 \\
\hline \multicolumn{4}{|l|}{ Estado civil: } \\
\hline casado & 55,59 & 61,90 & 60,63 \\
\hline \multirow{2}{*}{\multicolumn{4}{|c|}{ Género: }} \\
\hline & & & \\
\hline Hombre & 57,62 & 60,90 & 63,64 \\
\hline \multirow{2}{*}{\multicolumn{4}{|c|}{ Nivel educativo: }} \\
\hline & & & \\
\hline Hasta educación postsecundaria no superior & 64,68 & 72,96 & 83,65 \\
\hline $\begin{array}{r}\text { Educación superior } \\
\end{array}$ & 35,32 & 27,04 & 16,35 \\
\hline
\end{tabular}

Si observamos las características socioeconómicas de ambas clases, según su probabilidad de pertenencia a clase o grupo de preferencia, lo que se muestra en las Tablas 5 y $\mathbf{6}$, podemos apreciar que la clase 2 supera la renta mediana en el $54 \%$ de los casos frente al $46 \%$ de la clase 1; que la media de edad es algo más joven en la clase 2 con 57 años respecto de la clase 1 con 59 años; y que los hogares de la clase 2 experimentan en mayor porcentaje de casos los problemas ambientales asociados a las grandes ciudades como son el ruido, la contaminación y la delincuencia respecto a los hogares de la clase 1 . La clase 2 presenta mayor porcentaje de nivel educativo superior con el $33 \%$ de los hogares frente al 25\% de la clase 1 . Se observa en la clase 2 menor presencia de la mujer como responsable del hogar (35\%), menor frecuencia del tamaño de hogar más pequeño (73\%) y mayor porcentaje de hogares con niños (36\%) respecto de la clase 1 cuyos porcentajes respectivamente son el $45 \%$ (mujer), el 78\% (hogar más pequeño) y el 31\% (hogar con niños). Así pues, y siguiendo a Walter y Li (2007), distinguiríamos en este trabajo empírico dos estilos de vida asociados a cada clase o grupo de 
preferencia: uno, el suburbano, de menor renta, de menor nivel educativo, con mayor frecuencia del tamaño de hogar más pequeño, con mayor frecuencia de la mujer como cabeza de familia, y otro, el urbano, con mayor poder adquisitivo, con mayor nivel educativo, con más presencia del varón como responsable del hogar y con mayor frecuencia del tamaño de hogar superior a tres miembros.

Con respecto a los otros dos modelos estimados, se observa en la Tabla 3 que en el modelo mixto (MLM) la renta en zona rural, así como también, el hogar con niños en zona rural ${ }^{6}$ presentan un comportamiento aleatorio. La edad presenta el mismo patrón de comportamiento en todos los modelos estimados salvo para los hogares integrantes de la clase 2 del modelo LCCLM que no es significativa en la zona interurbana, y tiene un efecto positivo y significativo en la zona rural respecto de la zona urbana. En general, a mayor edad, la preferencia es la localización de la vivienda en zona urbana salvo la excepción comentada anteriormente. El patrón de comportamiento en cuanto a la edad es diferente en la clase 2 en la alternativa rural respecto de la zona urbana. La media de edad de los responsables del hogar integrados en la clase 2 es algo inferior respecto de los integrantes de la clase 1 (Tabla 5 y 6). Se puede deducir que los integrantes de la clase 2 están en un ciclo de vida diferente a los integrantes de la clase 1 que son por término medio de mayor edad por lo que esta situación afecta a la preferencia en cuanto a la elección de la zona de residencia. En general, la ausencia de contaminación y delincuencia inclinan la preferencia hacia la localización de la vivienda en la zona rural, y hacia la zona interurbana, aunque con menor impacto en este último caso, lo que también puede apreciarse en los efectos marginales del MNL (Tabla 4). El ruido no es significativo en la zona interurbana tanto en el MNL como en el MLM, así como, tampoco, en la clase 2 del LCCLM (Tabla 3). En la clase 1, el ruido en la zona interurbana es poco significativo.

Tabla 3: Resultados de la estimación_ECV_2017 (EU_SILC_2017). Alternativa de referencia: zona urbana

\begin{tabular}{|c|c|c|c|c|c|c|c|c|}
\hline \multirow{4}{*}{ Atributos } & \multirow{2}{*}{\multicolumn{2}{|c|}{ MNL }} & \multirow{2}{*}{\multicolumn{2}{|c|}{ MLM }} & \multicolumn{4}{|c|}{ LCCLM } \\
\hline & & & & & $\mathrm{Cla}$ & & Cla & \\
\hline & \multirow{2}{*}{$\begin{array}{c}\text { zona } \\
\text { interurbana }\end{array}$} & \multirow[t]{2}{*}{ zona rural } & $\begin{array}{c}\text { zona } \\
\text { interurbana }\end{array}$ & zona rural & \multirow{2}{*}{$\begin{array}{c}\text { zona } \\
\text { interurbana }\end{array}$} & \multirow[t]{2}{*}{ zona rural } & \multirow{2}{*}{$\begin{array}{c}\text { zona } \\
\text { interurbana }\end{array}$} & \multirow[t]{2}{*}{ zona rural } \\
\hline & & & \multicolumn{2}{|l|}{ media } & & & & \\
\hline Renta & $-0,249^{* * * *}$ & $-0,511^{* * *}$ & $-0,273^{* * *}$ & $-0,628^{* * * 1}$ & $-0,087^{* * * *}$ & $-0,255^{* * *}$ & $-0,396$ & $-0,941^{* * * *}$ \\
\hline Ruido & 0,094 & $0,330^{* * *}$ & 0,081 & $0,336^{* * *}$ & $0,501^{*}$ & $0,489^{* * *}$ & $-0,209$ & $0,200^{* * *}$ \\
\hline Contaminación & $0,354^{* * * *}$ & $0,563^{* * *}$ & $0,298^{* *}$ & $0,466^{* * *}$ & $1,063^{\text {**** }}$ & $1,708^{* * *}$ & $-0,199$ & $-0,614^{* * *}$ \\
\hline Delincuencia & $0,742^{* * *}$ & $1,179^{* * *}$ & $0,682^{* * *}$ & $1,138^{* * *}$ & $0,952^{* * *}$ & $1,563^{* * *}$ & 0,572 & $0,770^{* * *}$ \\
\hline $\begin{array}{l}\text { Tipo de } \\
\text { vivienda_piso }\end{array}$ & $-1,555^{* * *}$ & $-3,043^{* * *}$ & $-1,595^{* * *}$ & $-3,298^{* * *}$ & $-2,110^{* * *}$ & $-3,790^{* * *}$ & $-1,534$ & $-2,996^{* * *}$ \\
\hline $\begin{array}{l}\text { Nhabita*Tipo } \\
\text { vivienda }\end{array}$ & $0,171^{* *}$ & $0,326^{* * *}$ & $0,190^{* * *}$ & $0,343^{* * * *}$ & $-0,535$ & $0,419^{* * *}$ & $0,804^{* * *}$ & $0,035^{*}$ \\
\hline Edad & $-0,015^{* * *}$ & $-0,016^{* * *}$ & $-0,016^{* * *}$ & $-0,020^{* * *}$ & $-0,016^{* * *}$ & $-0,041^{* * *}$ & $-0,020$ & $0,006^{* * *}$ \\
\hline Hogar con niños & 0,130 & 0,068 & 0,035 & $-0.174^{* 2}$ & 0,086 & 0,153 & 0,024 & $-0,360^{* * *}$ \\
\hline Casado & $0,211^{* * * *}$ & $0,141^{*}$ & $0,186^{* * * *}$ & $0,095^{3}$ & 0,456 & $0,264^{* *}$ & 0,001 & $0,027^{* * * *}$ \\
\hline Nativo & 0,132 & $0,325^{* * *}$ & 0,081 & $0,280^{* *}$ & $-0,218$ & $0,413^{* * *}$ & 0,422 & $-0,061^{* * *}$ \\
\hline Tamaño hogar & $-0,007$ & 0,051 & $-0,060$ & $-0,048^{* * *}$ & $-0,443^{*}$ & 0,056 & 0,239 & $-0,395^{\text {*** }}$ \\
\hline Sexo & $-0,023$ & $-0,183^{* * *}$ & $-0,035$ & $-0,219^{* * *}$ & $-0,355$ & $-0,404^{* *}$ & 0,202 & $-0,084^{* * *}$ \\
\hline Nivel educativo & $0,471^{* * * *}$ & $0,987^{* * * *}$ & $0,452^{* * *}$ & $1,014^{* * * *}$ & $0,279^{* * *}$ & $0,900^{* * *}$ & 0,608 & 1,157 \\
\hline Constante & $-0,412^{*}$ & $-0,651^{* *}$ & \multicolumn{2}{|r|}{ 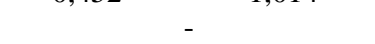 } & $49,90 \% \mathrm{de}$ & muestra & \multicolumn{2}{|c|}{$50,10 \%$ de la muestra } \\
\hline \multicolumn{3}{|c|}{ Log-likelihood $=-11.536,95$} & Log-likelihoo & $-11.533,24$ & & og-likelihor & $=-11.509,48$ & \\
\hline \multicolumn{3}{|c|}{ Pseudo- $\mathrm{R}^{2}=0,17$} & \multicolumn{2}{|c|}{$\rho^{2}(26)=0,21$} & \multicolumn{4}{|c|}{$\rho^{2}(26)=0,23$} \\
\hline
\end{tabular}

Se observa una menor preferencia de la localización de la vivienda en zona urbana en relación con estos problemas ambientales: ruido, contaminación atmosférica y delincuencia. No obstante, la clase 2 del LCCLM en la alternativa rural muestra un comportamiento diferente en relación con la contaminación atmosférica de forma que a pesar de experimentar con mayor frecuencia este tipo de problemas ambientales con el 95\% de los casos (ver Tabla 6) presenta una clara preferencia por la zona urbana.

\footnotetext{
${ }^{6}$ Los parámetros asociados a la renta en zona rural y hogares con niños en zona rural presentan una desviación típica significativa por lo que responden a un comportamiento aleatorio.
} 
Tabla 4: Efectos marginales de las variables en el MNL.

\begin{tabular}{lcccccc}
\hline \multirow{2}{*}{ Variables } & \multicolumn{3}{c}{ Efecto marginal medio } & \multicolumn{2}{c}{$\begin{array}{c}\text { Efecto marginal en el valor medio de las } \\
\text { variables de la muestra }\end{array}$} \\
\cline { 2 - 7 } & $\begin{array}{c}\text { Zona } \\
\text { urbana }\end{array}$ & $\begin{array}{c}\text { Zona } \\
\text { interurbana }\end{array}$ & $\begin{array}{c}\text { Zona } \\
\text { rural }\end{array}$ & $\begin{array}{c}\text { Zona } \\
\text { urbana }\end{array}$ & $\begin{array}{c}\text { Zona } \\
\text { interurbana }\end{array}$ & Zona rural \\
\hline Renta & 0,068 & $-0,009$ & -0.059 & 0,092 & $-0,0178$ & $-0,074$ \\
\hline Ruido & $-0,037$ & $-0,004$ & 0,042 & $-0,050$ & $-0,0010$ & 0,052 \\
\hline Contaminación & $-0,083$ & 0,023 & 0,059 & $-0,112$ & 0,0351 & 0,077 \\
\hline Delincuencia & $-0,175$ & 0,049 & 0,125 & $-0,236$ & 0,0735 & 0,162 \\
\hline Tipo de vivienda_piso & 0,413 & $-0,067$ & $-0,346$ & 0,561 & $-0,1207$ & $-0,440$ \\
\hline $\mathrm{N}^{\mathbf{o}}$ habitaciones por tipo vivienda & $-0,044$ & 0,008 & 0,036 & $-0,060$ & 0,0137 & 0,046 \\
\hline Edad & 0,003 & $-0,001$ & $-0,001$ & 0,004 & $-0,002$ & $-0,002$ \\
\hline Hogar con niños & $-0,019$ & 0,017 & 0,001 & $-0,025$ & 0,0209 & 0,004 \\
\hline Casado & $-0,033$ & 0,026 & 0,007 & $-0,044$ & 0,0324 & 0,012 \\
\hline Nativo & $-0,040$ & 0,001 & 0,039 & $-0,055$ & 0,006 & 0,049 \\
\hline Tamaño del hogar & $-0,003$ & $-0,004$ & 0,007 & $-0,005$ & 0,004 & 0,009 \\
\hline Sexo & 0,017 & 0,007 & $-0,025$ & 0,024 & 0,006 & $-0,030$ \\
\hline Educación & $-0,130$ & 0,016 & 0,114 & $-0,177$ & 0,033 & 0,145 \\
\hline
\end{tabular}

La preferencia por la residencia en piso y zona urbana es un patrón de comportamiento bastante estable en todas las estimaciones. Por el contrario, cuando se trata de una vivienda grande en cuanto al número de habitaciones ("Nhabita*Tipo vivienda"), la preferencia se inclina hacia las zonas interurbana y rural que es, por otra parte, donde hay mayor porcentaje de viviendas unifamiliares. En el MLM, la variable "hogar con niños" en el ámbito rural se ajusta mejor a un comportamiento aleatorio, como ya se ha comentado anteriormente. Esta heterogeneidad en el comportamiento de los hogares con niños también se observa entre las clases del LCCLM de forma que, si bien, en la clase 1 la variable "hogar con niños" no es significativa al igual que en MNL; sí es significativa en la clase 2 del LCCLM en el mismo sentido que en el MLM, inclinándose la preferencia hacia la zona urbana. El estado civil de "casado" presenta el mismo comportamiento en todos los modelos estimados con una clara preferencia por la zona interurbana y rural respecto de la zona urbana, aunque esta variable no es significativa en todos los casos. En cuanto al "nativo" la preferencia cuando la variable es significativa es hacia la zona rural, no siendo significativa la zona interurbana. No obstante, el "nativo" en la clase 2 del LCCLM manifiesta preferencia hacia la zona urbana. Recordemos también en este punto que la media de edad del responsable de hogar de la clase 2 es algo inferior respecto a los de la clase 1 lo que lo sitúa en un ciclo de vida diferente (Tablas 5 y 6 ).

Tabla 5: Edad media y probabilidad de elegir según la clase de pertenencia

\begin{tabular}{ccccc}
\hline Obs. & Clase & Media & S. D. & Probabilidad condicionada \\
\hline 5.421 & 1 & 60 & 15,39 & 0,70 \\
4.937 & 2 & 58 & 15,97 & 0,70 \\
2.299 & 1 & 59 & 17,11 & 0,80 \\
1.947 & 2 & 58 & 14,99 & 0,80 \\
820 & 1 & 60 & 15,42 & 0,90 \\
288 & 2 & 57 & 15,08 & 0,90 \\
\hline \multicolumn{4}{l}{ Nota: S.D.=Standard Deviation. }
\end{tabular}

En cuanto a la bondad del ajuste del LCCLM, una medida cuantitativa del adecuado ajuste del modelo en cuanto a la diferenciación de las clases según las preferencias consiste en calcular el promedio de la probabilidad posterior más alta respecto del total de la muestra (Pacifico et al., 2013: “cpmax"). Como puede observarse en la Tabla 7, el promedio para el conjunto de la muestra alcanza el valor de $61 \%$ lo que puede considerarse un ajuste bastante aceptable. 
Studies for Applied Economics Vol 37-3

Tabla 6: Características socioeconómicas según la clase de preferencia ${ }^{7}$

\begin{tabular}{|c|c|c|c|c|c|c|c|c|}
\hline \multirow{2}{*}{ Variable } & \multicolumn{2}{|c|}{ Media } & \multicolumn{2}{|c|}{ Desviación típica } & \multicolumn{2}{|c|}{ Min } & \multicolumn{2}{|c|}{ Max } \\
\hline & Clase_1 & Clase_2 & Clase_1 & Clase_2 & Clase_1 & Clase_2 & Clase_1 & Clase_2 \\
\hline Renta & 0,46 & 0,54 & 0,006 & 0,006 & 0,45 & 0,53 & 0,47 & 0,55 \\
\hline Ruido & 0,82 & 0,89 & 0,004 & 0,003 & 0,81 & 0,88 & 0,83 & 0,89 \\
\hline Contaminación & 0,88 & 0,95 & 0,004 & 0,002 & 0,87 & 0,95 & 0,89 & 0,96 \\
\hline Delincuencia & 0,89 & 0,94 & 0,004 & 0,003 & 0,88 & 0,93 & 0,89 & 0,94 \\
\hline Tipo vivienda_piso & 0,60 & 0,72 & 0,006 & 0,005 & 0,59 & 0,71 & 0,61 & 0,73 \\
\hline $\mathrm{N}^{\circ}$ habs. *tipo de vivienda & 0,66 & 0,60 & 0,005 & 0,005 & 0,65 & 0,58 & 0,67 & 0,61 \\
\hline Edad & 59,38 & 57,25 & 15,24 & 15,87 & 17 & 18 & 86 & 86 \\
\hline Hogar con niños & 0,31 & 0,36 & 0,005 & 0,005 & 0,30 & 0,35 & 0,32 & 0,37 \\
\hline Casado & 0,54 & 0,62 & 0,006 & 0,005 & 0,53 & 0,61 & 0,55 & 0,63 \\
\hline Nativo & 0,92 & 0,90 & 0,003 & 0,003 & 0,91 & 0,89 & 0,92 & 0,91 \\
\hline Tamaño hogar & 0,78 & 0,73 & 0,005 & 0,005 & 0,77 & 0,74 & 0,79 & 0,76 \\
\hline Sexo & 0,45 & 0,35 & 0,006 & 0,005 & 0,44 & 0,34 & 0,46 & 0,36 \\
\hline Nivel educativo & 0,75 & 0,67 & 0,005 & 0,005 & 0,74 & 0,66 & 0,76 & 0,69 \\
\hline Observaciones & 6.684 & 6,701 & 6.684 & 6,701 & 6.684 & 6,701 & 6.684 & 6,701 \\
\hline
\end{tabular}

Tabla 7: Ajuste del modelo a las clases de preferencias

\begin{tabular}{|c|c|c|c|c|c|}
\hline Indicador & Obs. & Media & $\begin{array}{c}\text { Desviación } \\
\text { típica }\end{array}$ & Min & Max \\
\hline cpmax & 13.430 & 0,61 & 0,10 & 0,50 & 0,98 \\
\hline
\end{tabular}

\section{CONCLUSIONES}

Los resultados obtenidos del análisis empírico realizado prueban que en una misma muestra de hogares se pueden distinguir diferentes grupos o clases de preferencias en relación con la localización de la vivienda. Así pues, se aprecian dos grupos o clases de preferencias que se corresponden con dos estilos de vida o comportamiento diferentes cuales son: uno, el suburbano integrado por hogares de menor renta, menor nivel educativo, mayor frecuencia del tamaño de hogar más pequeño, mayor frecuencia de la mujer como cabeza de familia, y por otro, el urbano integrado por hogares con mayor poder adquisitivo, mayor nivel educativo, con más presencia del varón como cabeza de familia y con mayor frecuencia del tamaño de hogar superior a tres miembros. El modelo de clases latentes nos permite observar con mayor profundidad la heterogeneidad en el comportamiento de los hogares con respecto a la elección de la localización de la vivienda. El modelo de clases latentes nos proporciona información sobre la probabilidad de un hogar de pertenecer a un determinado grupo o clase, así como, también, nos proporciona información respecto a su elección. El impacto de las características observables en la elección de la localización de la vivienda no siempre se mueve en la misma dirección. Dependerá, entre otros aspectos, de las preferencias del grupo o clase a la que pertenezca el hogar la cual se asocia a un determinado estilo de vida. Este es un resultado con mayor potencialidad de análisis respecto de los tradicionales modelos multinomial y mixto que con mayor frecuencia se aplican en relación con el análisis de la elección de vivienda. Así, por ejemplo, se observa que la preferencia respecto a los factores ambientales como son el ruido o la delincuencia tiene el mismo patrón de comportamiento en todos los modelos estimados cuando la variable es significativa. No obstante, no sucede lo mismo con la contaminación atmosférica de forma que los integrantes de la clase 2 del modelo de clases latentes de mayor poder adquisitivo, por cuanto superan la renta mediana con mayor frecuencia (54\%), siguen prefiriendo la zona urbana a pesar de sufrir con mayor porcentaje de hogares $(95 \%)$ el problema de la contaminación atmosférica. Este grupo de hogares o clase de preferencias es de menor edad media y mayor nivel educativo que respecto de la clase 1 . Se observa, por otra parte, que la clase 2 no manifiesta preferencia por la zona interurbana.

No obstante, con respecto a la renta no hay duda, que la preferencia es hacia una mayor concentración de los hogares en la zona urbana. A mayor renta, la preferencia es hacia la localización de la vivienda en zona urbana. El mismo resultado se obtiene respecto de la edad de forma que a mayor edad, la preferencia se inclina hacia la zona urbana a excepción de la clase 2 del modelo latente

\footnotetext{
${ }^{7}$ Probabilidad posterior a la estimación superior al $50 \%$.
} 
(LCCLM) que, como ya ha sido comentado a lo largo de este trabajo, al ser un grupo de menor edad media respecto a la clase 1 , es posible que su situación con respecto al ciclo de vida afecte a las preferencias de localización. Esta es una de las conclusiones más significativas del análisis pues según el ciclo de vida en el que se encuentre el hogar medido a través del responsable del hogar, el impacto de las variables socioeconómicas que les caracterizan es diferente. En el mismo sentido, es interesante destacar como resultado del modelo mixto (MLM) que los "hogares con niños" presentan un patrón de comportamiento aleatorio. En el modelo latente (LCCLM) se observa que, si bien para la clase 2, la preferencia es la misma que en el modelo mixto (MLM), zona urbana; sin embargo, en la clase 1 del modelo latente, la variable no es significativa. En este último caso (clase 1), hay menor frecuencia de hogares con niños (31\%) respecto de la clase 2 (36\%), teniendo en cuenta la probabilidad de pertenencia a clase posterior a la estimación del modelo latente (prob.>0,50). No hay duda que las preferencias con respecto a los estilos de vida según sea la zona rural, urbana o interurbana junto con el ciclo de vida, las características socioeconómicas de los hogares, así como los atributos específicos de la alternativa, inciden en la decisión en cuanto a la localización de la residencia lo cual puede observarse a partir de las elecciones realizadas. Esto es, a partir de las preferencias reveladas.

\section{REFERENCES}

BARRIOS GARCÍA J. Y J. E. RODRÍGUEZ (2007): "Housing and urban location decisions in Spain: An econometric analysis with unobserved heterogeneity". Urban Studies, 44 (9): 1657-1676.

BARRIOS GARCÍA J. Y J. E. RODRÍGUEZ (2008): "Housing demand in Spain according to dwelling type: microeconometric evidence". Regional Science and Urban Economics 38: 363-377.

BEER A., D. FAULKNER, C. PARIS Y T. CLOWER (2011): Housing Transitions Through the Life Course; aspirations, needs and policy. Bristol: The Policy Press.

BELL D. Y A. RUTHERFORD (2012): Long-term care and the housing market. Scottish Journal of Political Economy 59 (5): 543-563.

BOURASSA S. C. (1995): "A model of housing tenure choice in Australia". Journal of Urban Economics 37: 161175.

BOVER O. Y P. VELILLA (2001): "Precios hedónicos de la vivienda sin características: el caso de las promociones nuevas". Banco de España. Estudios Económicos nำ73.

BRAMLEY G. y D. WATKINS (2016): Housebuilding, demographic change and affordability as outcomes of local planning decisions: exploring interactions using a sub-regional model of housing markets in England. Progress in Planning 104: 1-35.

BROWN J. N. y H. S. ROSEN (1982): "On the estimation of structural hedonic price models". Econometrica 50 (3): $765-768$.

BUZAR S., P. OGDEN, R. HALL, A. HAASE, S. KABISCH y A. STEINFIIHRER (2007): Splintering urban populations: emergent landscapes of reurbanisation in four Europeans cities. Urban Studies 44 (4): 651 677.

CASTLES F. (1998): The really big trade-off: home ownership and the welfare state in the new world and the old. Acta Politica 33(1): 5-19.

CLAPHAM D. (2018): "Housing Theory, Housing Research and Housing Policy". Housing, Theory and Society 35 (2): 163-177.

COLOM ANDRÉS M. C. y M. C. MOLÉS MACHÍ (1999): “Una aproximación a los condicionantes de la elección de vivienda secundaria”. Estudios de Economía Aplicada, 11:23-39.

DEWILDE C. (2008): Divorce and the housing movements of owner-occupiers: A European comparison. Housing Studies 23(6): 809-832.

DILLON WR, A. KUMAR A y MS BORRERO (1993): "Capturing individual differences in paired comparisons: an extended BTL Model incorporating descriptor variables". Journal of Marketing Research 30(1):42-51

DURA-GUIMERA A. (2003): Population deconcentration and social restructuring in Barcelona, a European Mediterranean city. Cities 20: 387-394.

EICHHOLTZ P. y T. LINDENTHAL (2014): "Demographics, human capital, and the demand for housing". Journal of Housing Economics 26: 19-32.

ERMISCH J. (1996): The demand for housing in Britain and population ageing: microeconmic evidence. Economica 63: 383-404.

FAUBERT V., É. MONNET y C. SUTTER (2015): Despite the recovery of purchasing power, housing construction should keep falling in 2015. Notes de conjoncture. INSEE.

GALSTER G., R. ANDERSSON y S. MUSTERED (2010): "Who is affected by neighbourhoood income mix?. Gender, age, family, employment and income differences". Urban Studies 47 (1): 167-168.

GARCÍA J. y J. M. RAYA (2011): "Price and income elasticities of demand for housing characteristics in the city of Barcelona". Regional Studies 45 (5): 597-608.

GONZÁLEZ L. y F. ORTEGA (2013): "Immigration and housing booms: evidence from Spain". Journal of Regional Science 53 (1):37-59. 
GROVER R y V. SRINIVASAN (1987): "A simultaneous approach to market segmentation and market structuring". Journal of Marketing Research 24: 139-153.

HENDERSHOTT P. H, R. ONG, G. A. WOOD y P. FLATAU (2009): Marital history and home ownership: evidence from Australia. Journal of Housing Economics 18(1): 13-24.

HERNÁNDEZ ALEMÁN A. y C. J. LEÓN (2018): "Transición demográfica y demanda de vivienda en España. Explorando interacciones". Revista Galega de Economía, vol. 27 (2).

HESS, S., (2014): Latent class structures: taste heterogeneity and beyond, in Hess, S. y Daly, A.J. (Eds.), Handbook of Choice Modelling, chapter 14, pp. 311-330, Edward Elgar publishers, Cheltenham.

HESS, S., M. BEN-AKIVA, D. GOPINATH y J. WALKER (2011). "Advantages of latent class over continuous mixture of logit models". http://www.stephanehess.me.uk/papers/Hess Ben-Akiva Gopinath Walker May 2011.pdf.

KING P. (2009): "Using Theory or Making Theory: Can there be Theories of Housing?". Housing, Theory and Society 26 (1): 41-52.

LANCASTER K. J. (1966): "A New Approach to Consumer Theory". Journal of Political Economy 74: 132-157.

LEE T. H (1963): "Demand for housing: a cross-section analysis". The Review of Economics and Statistics 45 (2): 190-196.

LEISHMAN C. (2009): "Spatial change and the structure of urban housing sub-markets". Housing Studies 24 (5): 563-585.

MANKIW, G.N. y WEIL, D.N., (1989): "The baby boom, the baby bust, and the housing market”. Regional Science and Urban Economics 19 (2): 235-258.

MCFADDEN D. (1978): Modelling the choice of residential location, in A. Karlqvist A., L. Lundqvist, F. Snickars, and J. Weibull (eds.), Spatial Interaction Theory and Planning Models, pp.: 75-96. North Holland: Amsterdam.

MCFADDEN D. y K. TRAIN (2000): "Mixed MNL models for discrete response". Journal of Applied Economics 15 (5): 447-470

MEEN G. (2016): "Spatial housing economics: a survey”. Urban Studies 53(10):1987-2003.

MONNET E. y C. WOLF (2017): "Demographic cycles, migration and housing investment". Journal of Housing Economics 38: 38-49.

MYERS D. (1990): Housing Demography : Linking Demographic Structure and Housing Markets. Edited by Dowell Myers. University of Wisconsin Press Madison, Wis.

OGDEN P. y R. HALL (2000): Households, reurbanisation and the rise of living alone in the principal French cities 1975-1900. Urban Studies 37: 367-390.

ONG R., G. WOOD y V. COLIC-PEISKER (2015): Housing older Australians: Loss of homeownership and pathways into housing assistance. Urban Studies 52(16):2979-3000.

OTTENSMANN J. R., S. PAYTON y J. MAN (2008): "Urban location and housing prices within a hedonic model". The Journal of Regional Analysis and Policy 38 (1): 19-35.

PACÍFICO D. y H. IL YOO (2013): "Lclogit: A Stata command for fitting latent-class conditional logit models via the expectation-maximization algorithm". The Stata Journal 13 (3): 625-639.

PERSSON T. H. (2002): "Welfare calculations in models of the demand for sanitation". Applied Economics 34: 1509-18.

RODRIGUEZ HERNANDEZ J.E. y J. A. BARRIOS GARCÍA (2003): "Un modelo logit mixto de tenencia de vivienda en Canarias". Estudios de Economía Aplicada, 21 (1), pp. 175-191.

RODRIGUEZ HERNANDEZ J.E. y J. A. BARRIOS GARCÍA (2007): "Estimación microeconométrica de la tenencia y demanda de vivienda en España según la localización”. Estudios de Economía Aplicada, 25 (1):453-484

SHEN J. (2009): "Latent class model or mixed logit model?. A comparison by transport mode choice data". Applied Economics 41: 2915-2924.

SMITH T R., MERTZ F. (1980): "An analysis of the effects of information revision on the outcome of housing market search, with special reference to the influence of realty agents". Environment and Planning A 12: 155-174.

SWAIT J y JC SWEENEY (2000): "Perceived value and its impact on choice behavior in a retail setting". Journal of Retailing and Consumer Services 7 (2):77-88

TRAIN K. E. (2003): Discrete choice models with simulation. Cambridge University Press, Cambridge

WALKER J. y J. LI (2007): "Latent lifestyle preferences and household location decisions". Journal of Geographical Systems 9 (1): 77-101.

WONG G. K. (2002): "A conceptual model of the household's housing decisión-making process: the economic perspective". Regional of Urban \& Regional Development Studies 14 (3): 217-234.

ZHANG J. (2004): "A dynamic model of residential segregation”. Journal of Mathematical Sociology 28:147-170. 


\section{Apéndice}

Tabla A1.1: Definición de las variables empleadas en los modelos estimados

\begin{tabular}{|c|c|}
\hline Variable & Definición \\
\hline Renta_hogar ${ }^{8}$ & $\begin{array}{l}=1 \text { renta por encima de la mediana de la encuesta. Desde } \\
24.107 € / \text { año hasta } 343.118 € / \text { año. } \\
=0 \text { cero en cualquier otro caso }\end{array}$ \\
\hline Ruido & $\begin{array}{l}\text { = } 1 \text { si la vivienda no está afectada por problemas de ruidos } \\
\text { producidos por vecinos o procedentes del exterior (tráfico, } \\
\text { negocios, fábricas, etc.); } \\
=0 \text { cero en cualquier otro caso. }\end{array}$ \\
\hline Contaminación & $\begin{array}{l}=1 \text { si la vivienda no está afectada por la contaminación } \\
\text { derivada de la industria y el tráfico; } \\
=0 \text { cero en cualquier otro caso. }\end{array}$ \\
\hline Delincuencia & $\begin{array}{l}=1 \text { si la vivienda no está afectada por problemas de } \\
\text { delincuencia o vandalismo en la zona; } \\
=0 \text { cero en cualquier otro caso. }\end{array}$ \\
\hline Tipo de vivienda_piso & $\begin{array}{l}=1 \text { si se trata de una residencia en piso; }=0 \text { en otro caso } \\
\text { (unifamiliar). }\end{array}$ \\
\hline Edad & $\begin{array}{l}\text { Se corresponde con la edad media del cabeza de familia o } \\
\text { responsable del hogar. Se considera responsable de la } \\
\text { vivienda a aquel miembro del hogar a cuyo nombre está el } \\
\text { título de propiedad o el contrato de arrendamiento o } \\
\text { subarrendamiento de la vivienda en alquiler. En el caso de } \\
\text { que el hogar disfrute de la vivienda por cesión gratuita, se } \\
\text { considerará responsable de la vivienda al miembro del } \\
\text { hogar a quien se le haya cedido. }\end{array}$ \\
\hline $\mathrm{N}^{\circ}$ habitaciones por tipo vivienda & $\begin{array}{l}\text { Es la combinación de dos variables: número de habitaciones } \\
\text { que toma el valor } 1 \text { para las viviendas con cinco o más } \\
\text { habitaciones, y cero en otro caso, multiplicado por la } \\
\text { tipología de la vivienda que toma el valor } 1 \text { si se trata de } \\
\text { piso, y cero cuando es unifamiliar. }\end{array}$ \\
\hline Hogar con niños & $\begin{array}{l}=1 \text { para aquellos hogares que tengan niños (hijos } \\
\text { dependientes económicamente) })^{9} ; \\
=0 \text { en cualquier otro caso. }\end{array}$ \\
\hline Casado & $\begin{array}{l}=1 \text { si el responsable del hogar es casado; } \\
=0 \text { en cualquier otro caso. }\end{array}$ \\
\hline Nativo & $\begin{array}{l}=1 \text { si el responsable es español } \\
=0 \text { en cualquier otro caso. }\end{array}$ \\
\hline Tamaño hogar & $\begin{array}{l}=1 \text { si el hogar es de menor tamaño. Entre } 1 \text { y tres } \\
\text { miembros; } \\
=0 \text { en cualquier otro caso. Entre } 4 \text { y } 13 \text { miembros. }\end{array}$ \\
\hline Sexo & $=1$ si el cabeza de familia es mujer; $=0$ en otro caso. \\
\hline
\end{tabular}

\footnotetext{
${ }^{8}$ La renta disponible total del hogar se calcula de la siguiente manera: + Renta bruta monetaria o cuasimonetaria del asalariado + Renta bruta no monetaria del asalariado + Cotizaciones sociales a cargo del empleador + Beneficios o pérdidas monetarios brutos de trabajadores por cuenta propia (incluidos derechos de propiedad intelectual) + Valor de los bienes producidos para autoconsumo + Prestaciones por desempleo + Prestaciones por vejez + Prestaciones por supervivencia + Prestaciones por enfermedad + Prestaciones por invalidez + Ayudas para estudios + Ayudas por familia/hijos + Exclusión social no clasificada en otro apartado + Ayudas para vivienda + Alquiler imputado + Renta procedente del alquiler de una propiedad o terreno + Intereses, dividendos y ganancias de inversiones de capital en empresas no constituidas en sociedad + Transferencias periódicas monetarias percibidas de otros hogares + Renta percibida por los menores de 16 años - Intereses pagados de préstamos hipotecarios - Cotizaciones sociales a cargo del empleador - Impuesto sobre la renta y cotizaciones sociales (incluye devoluciones/ingresos complementarios por ajustes en impuestos sobre la renta, impuesto sobre la renta retenido en origen y cotizaciones sociales -si procede) - Impuesto sobre el patrimonio - Transferencias periódicas monetarias pagadas entre hogares.
}

${ }^{9}$ Son los supuestos 10, 11, 12, 13 y 14 de la variable "Tipo de Hogar" de la Encuesta de Condiciones de vida (HX060). 
Nivel educativo

Alternativa 1: Zona urbana*

Alternativa 2: Zona interurbana*
$=1$ si el nivel educativo del cabeza de familia es inferior a la educación postsecundaria no superior. Esto es, menos que primaria, educación primaria, primera y segunda etapa de la educación secundaria hasta la educación postsecundaria no superior; $=0$ en otro caso (educación superior).

Cuando la vivienda del hogar está ubicada en zona muy poblada. Se define como una zona densamente poblada. Conjunto contiguo de unidades locales, cada una de las cuales con una densidad de más de 500 habitantes por $\mathrm{km}$ cuadrado y con una población total de al menos 50.000 habitantes.

Cuando la vivienda del hogar está ubicada en zona medio poblada. Se define como una zona semiurbana o intermedia. Conjunto contiguo de unidades locales, no pertenecientes a una zona densamente poblada, donde cada una tiene una densidad superior a los 100 habitantes por km cuadrado y donde la población total es al menos de 50.000 habitantes o es adyacente a una zona densamente poblada. Es la variable DB100 de la Encuesta de Condiciones de Vida.

Alternativa 3: Zona rural ${ }^{*}$

Cuando la vivienda del hogar está ubicada en zona poco poblada. Se define como una zona escasamente poblada. Conjunto contiguo de unidades locales, no formando ni una zona densamente poblada ni una zona intermedia. Es la variable DB100 de la Encuesta de Condiciones de Vida.

${ }^{*}$ Metodología de la Encuesta de Condiciones de Vida. Datos básicos del hogar. Ine. 